\title{
Year in Review 2020: Nutrition and Gastrointestinal Disease in Cystic Fibrosis
}

\author{
Meghana Sathe ${ }^{1}$, Preeti Sharma ${ }^{2}$, and Adrienne Savant ${ }^{3}$ \\ ${ }^{1}$ University of Texas Southwestern Medical Center at Dallas \\ ${ }^{2}$ The University of Texas Southwestern Medical Center \\ ${ }^{3}$ Tulane University School of Medicine
}

June 4, 2021

\begin{abstract}
The multisystemic manifestations of cystic fibrosis (CF) involve all parts of the gastrointestinal (GI) system, including the pancreas, intestine and liver. As providers who care for people with $\mathrm{CF}(\mathrm{PwCF})$, knowledge of the manifestations, treatment and research related to nutrition and GI disease is important. This review is last installment of the CF Year in Review 2020 series, focusing on the multisystem effects of CF. Part one focused on the literature related to CFTR (cystic fibrosis transmembrane conductance regulator protein) modulators, while part two focused on pulmonary outcomes, radiographic and physiologic assessments, as well as infection and inflammation. Part three was split into Part 3A, focusing on the multisystem impact of $\mathrm{CF}$, and this review, Part 3B, focusing on nutritional, gastrointestinal and hepatobiliary articles. Articles were chosen from Pediatric Pulmonology but also include articles published in 2020 from other journals that are of particular interest to clinicians.
\end{abstract}

\section{Introduction:}

Although advances in cystic fibrosis (CF) care with the development, availability, and use of CF transmembrane regulatory conductance channel (CFTR) modulators have changed the disease trajectory, there continues to be a need for increased understanding related to nutrition and gastrointestinal disease for people with $\mathrm{CF}(\mathrm{PwCF})$. Nutrition has always been a cornerstone of CF care due to pancreatic insufficiency (PI) and the need for pancreatic enzyme replacement therapy (PERT), however many questions continue to remain unanswered. Pancreatic insufficiency can lead to decreased fat absorption (even with PERT) and reduced nutrient intake, thus understanding optimal PERT doing, use of supplements and knowledge regarding deficiencies can help improve overall care. Gastrointestinal health goes beyond nutrition to include the microbiome, an area of rapidly expanding research that is linked to alterations in nutritional outcomes. In addition, liver disease in CF is currently being redefined with recent longitudinal studies and advances in technology adding to our understanding. This review will cover publications related to gastrointestinal health, including PERT dosing, nutritional deficiencies and new supplements, intestinal microbiome, and liver disease.

\section{NUTRITION}

Nutritional management of CF starts with determination of pancreatic status (PI or PS). Poli et al. ${ }^{1}$ describes infants with CF who, despite having PI levels of fecal elastase at diagnoses, and thus starting pancreatic enzyme replacement therapy (PERT), were found to have normal fecal elastase (FE) levels at 2, 4, 5 and 6 years of age. The authors bring to light the importance of evaluating both $\mathrm{CF}$ genotype and fecal elastase (FE) measurements together in context of genotypic norms. All of the genotypes $(\mathrm{F} 508 \mathrm{del} / 2789+5 \mathrm{G}>$ 
A, F508del/R1066H, and M1T homozygous) were shown to have low levels of PI on review of databases or review of the medical literature.

If patients with $\mathrm{CF}$ are found to be PI, the optimal dosing for PERT is still up for debate and an area for further research. The MyCyFAPP has been developed to help with self-management of gastrointestinal concerns $^{2}$. The app facilitates dosing of PERT via an algorithm that assigns optimal PERT doses based on food groups. Doses ranged from 1000-4000 lipase units/gram of fat and were based on a prior in vitro $\mathrm{CF}$ digestion model. Additionally, the MyCyFAPP, had an abdominal symptom diary, links to online tools, and educational modules. In a 6-month prospective, multicenter study of 148 patients, between the ages of 24 months and 18 years, use of MyCyFAPP was shown to improve quality life as measured on the CF PedsQL-GI. Patients $>12$ years of age were able to participate in the study independently. Patients had statistically significant improvements on the CF PedsQL-GI at 6 months, but not at three months, while parents reporting for their children [?]12 years of age had improvements at 3 months which were sustained through 6 months. Both patients and parents reported that they would want to use the app in their daily lives independent of the study. To specifically evaluate the PERT dosing algorithm, 58 patients had measurement of coefficient of fat absorption (CFA) at baseline and after use of the MyCyFAPP ${ }^{3}$. Although the CFA remained unchanged, the range of PERT dosing was reduced (1447-3070 LU/g fat versus 1783-2495 LU/g fat). Those with a low CFA ([?]90\%) at baseline had a significant improvement with the MyCyFAPP dosing, from $86.3 \%$ to $94 \%$ ( $\mathrm{p}=0.031$ ), due to improved adherence to recommended PERT dosing with consistent use of the app during the study period. However, it is difficult to determine if improvement in CFA was due to alternative dosing recommended by MyCyFAPP alone or simply improved adherence to PERT. Based on the results of the study, the authors suggest increasing the PERT dosing range to 1000-4000 LU/g of fat but recognize that longer periods of observation with more subjects are required to confirm results and make universal recommendations.

Another study looking at fat absorption, evaluated a novel nutritional supplement, Encala. Encala is a readily absorbable structured lipid made of a backbone of lysophosphatidylcholine (LPC) which optimizes dietary fatty acid absorption in the small intestine, enhances transfer of fatty acid into the lymphatic system and improves fatty acid retention in the mucosa ${ }^{4}$. Encala enhances absorption of long-chain triglycerides and the two essential fatty acids, linoleic and linolenic acid ${ }^{5}$. This unique supplement is a taste-neutral powder that is mixed with foods and beverages, is water soluble, does not require lipase for digestion/absorption, and has previously shown safety and tolerability. The study of Encala effect on baseline CFA was undertaken in children with $\mathrm{CF}$ age 5-17.9 years of age in a double-blind placebo trial with 1:1 randomization. Patients with CF who had a low CFA $(<88 \%)$ at baseline were found to have improved CFA $(78.9 \pm 7.5 \%$ to $86.3 \pm 6.7 \%$, $\mathrm{p}=0.002$ ), a decrease in stool fat loss and no change in dietary fat intake, while the high CFA group had no changes in the same parameters. In addition, patients with low CFA showed significant improvements in total plasma fatty acid concentration (20\%, p[?]0.005) and anthropometrics (height-for-age z-score (0.06 $+-0.08)$, weight-for-age z-score $(0.17+-0.16)$, and BMI z-score $(0.20+-0.25)$ with increases in all parameters (p[?]0.002) over a 3-month period. The data is encouraging for the use of Encala to optimize fat absorption above and beyond the capabilities of PERT.

Although the nutrition focus for $\mathrm{CF}$ is often on malnutrition due to malabsorption, other concerns are also being studied, such as micronutrient deficiency and the emerging concern of obesity. A cross sectional study (the Dietary Intake Study in cHildren (DISH), evaluated the dietary intake of 82 Australian children with CF compared to 82 healthy age and sex matched controls ${ }^{6}$. Children with CF consume more calories/energy, with increased absolute consumption of all micronutrients, except Vitamin $\mathrm{C}$ and folate. When adjusted for energy intake, CF children consumed less micronutrients per $1000 \mathrm{kcal}$ except in Vitamin A, sodium, calcium and phosphorus. A strong relationship with age and suboptimal micronutrient intake was seen, such that by high school, children with CF failed to meet the recommended daily intake amount for most key micronutrients.

Harindhanavudhi et al. ${ }^{7}$ evaluated the prevalence and factors associated with overweight and obese adults with CF at the University of Minnesota between January 2015-January 2017. Out of 484 adults, $25.6 \%$ were 
found to be overweight and $6.6 \%$ were obese. Specifically, $25 \%$ of those with a severe genotype (defined based on gene expression or protein function), were overweight/obese. Of those who were overweight/obese, there was a higher prevalence of hypertension $(31 \% / 25 \%$ versus $17 \%, \mathrm{p}=0.01)$, higher total cholesterol, LDL-cholesterol, and triglycerides, when compared to normal weight CF adults. Lung function was higher in overweight/obese patients ( $78-81 \%$ versus $59 \%$ [underweight] $70 \%$ [normal weight], p $<0.0001$ ), with a threshold effect beyond a BMI of $28-29 \mathrm{~kg} / \mathrm{m} 2$ whereby no further increases in FEV1 were seen.

\section{INTESTINE}

Case reports can be instructional and thought provoking for clinicians when they are presented with similar scenarios. Several case reports published this year are outlined in Table 1 with specific lessons learned highlighted.

The gut microbiome is defined as the diverse collection of microbial cells, their genetic material and the surrounding gut environmental conditions. The microbiome is an active participant in host physiology including energy metabolism, establishment and maintenance of the intestinal barrier, and immune system development. The microbiome undergoes changes over time, influenced by age, immune maturation, and environmental factors like diet, exercise, and antibiotic exposure. Dysbiosis occurs when there are significant changes to the composition of the microbiome in an individual relative to healthy individuals. Dysbiosis can lead to the development of diseases. For a growing number of diseases, an altered microbiome is not just a marker of disease, but also actively contributes to pathology. Not only are the number of microbes present important, the diversity or variety of species composition also accounts for the function of the gut microbial community $^{12}$. In practice, diversity is difficult to assess quantitatively, and several diversity measures have been adopted: alpha diversity is often estimated as the number of species in a community (species richness), and beta diversity is often based on the number of shared species among communities ${ }^{13}$.

Understanding the natural history of the microbiome over time is important. Only then can we begin to understand how environmental factors can impact the microbiome and subsequently how we can improve the dysbiosis created. Kristensen et al. ${ }^{14}$ studied 20 infants with CF during the first 18 months of life and showed that microbial development of the gut is different in healthy infants with CF compared to healthy infants without CF. While all infants initially seem to express a Bifidobacterium dominated profile, the microbiome in $\mathrm{CF}$ infants further deviates over time. Bifidobacterium has been shown to be associated with a range of beneficial health effects including the regulation of intestinal microbial homeostasis, the inhibition of pathogens and modulation of local and systemic immune responses. Over time, environmental factor influence changes in the gut microbiome including breastfeeding, exposure to transient bacteria through daycare exposure/older siblings, and antibiotic exposure. In this study, only infants with CF were observed to have significant antibiotic exposure in the first 18 months of life. Stools were collected monthly till 6 months of age and then at $8,10,12,15$ and 18 months of age. Over time, infants with CF were more likely to be colonized with pathogenic, proinflammatory bacteria such asStreptococci and Escherichia coli while control infants were more likely to be colonized with healthy bacteria such asAkkermansia and Anaerostipes spp .

Microbial community alterations through natural history or based on treatment/environmental factors contribute to CF pathophysiology. Antibiotic use in infants with CF further worsens this dysbiosis leading to a lower alpha diversity (decreased number of species), a further reduced abundance of Bifidobacterium and Bacteroides and a higher abundance of pathogenic bacteria, such as the Enterococcusgenus ${ }^{15}$. Khalaf et al. evaluated the role of acid blockade in development of pulmonary infections, debunking the previous presumption by showing that there is no influence of acid blockade on the microbiome of the aerodigestive tract. They evaluated respiratory (nasal and pulmonary) and gastrointestinal (oropharyngeal, esophageal, gastric and duodenal) samples in 25 children with $\mathrm{CF}$, on and off acid blockade. There was no significant difference between alpha diversity, beta diversity, total bacterial load and the presence of inflammation (increased IL-8 levels) between those on acid blockade and those not on acid blockage. In addition, there was no significant difference in distribution between different anatomic locations, except for the nares. The nares had a distinctly lower bacterial load, lower alpha diversity and a different beta diversity. The authors 
conclude that acid blockade does not play a significant role in the microbiome of the aerodigestive tract.

Another important exposure that has been speculated to influence the gut microbiome is secondhand smoke exposure (SHSe). Loman et al. ${ }^{16}$ recruited a group of children with CF (as defined by two-disease causing mutations and sweat chloride $>/=60 \mathrm{mmol} / \mathrm{L}$ ) ages 3 months to 5 years of age and analyzed clinical questionnaire, nicotine content of hair, oropharyngeal/respiratory samples, and fecal samples, noting xposure to antibiotics within 1 month of collecti. Results showed that SHSe was associated with an increase in Akkermansia, which is similarly observed in primary smokers and healthy infants without CF. Interestingly, Akkermansia is a mucin-degrading bacterium that is important in maintaining the integrity of the gastrointestinal tract. Decreased alpha diversity and a decrease in abundance of other beneficial bacterium, Bifidobacterium and Lactobacillusspecies, was seen independent of SHSe, but potentially related to antibiotic exposure. However, this causal relationship could not be determined on the basis of this study due to the relatively small number of participants. Loman et al. concluded that age, not exposure to SHSe or antibiotics, was the predominant factor in determination of microbiome composition in cohort of children with $\mathrm{CF}$.

The alterations in the microbiome are starting to be linked to clinical outcomes, especially in relation to growth. Analysis of the BONUS (Baby Observational and Nutrition Study) identified an early, progressive dysbiosis (decrease of Bacteroidetes and increase of Proteobacteria) distinguishing CF infants with low length from those with length ${ }^{17}$. Hayden et al. identified an early progressive dysbiosis in infants with $\mathrm{CF}$ and low length compared to infants with $\mathrm{CF}$ and normal length, including altered abundance of taxa associated with functions important for nutrient harvest and growth hormone signaling. They speculate that low length in infants with CF correlates with lower serum levels of IGF (insulin-like growth factors)-family of growth factors which may be modulated by the GI microbiota through short chain fatty acid (SCFA) production. SCFA are produced when gut microbiota ferment fiber and are known to induce IGF-1 which plays an important role in bone health and growth in infants and children. Based on this speculation, manipulation of the gut microbiome in infants with CF could potentially improve endocrine function impacting growth, especially length.

Beyond the gut microbiome, the importance of the intestinal virome has been more recently recognized. Coffey et al. ${ }^{18}$ provided the first description of the intestinal virome in children with $\mathrm{CF}$ comparing it to age matched control and finding differences in taxonomy. In children with CF, Picornavirales ,Picornaviridae , and Enterovirus positively correlated with weight and BMI, suggesting that the intestinal virome also contributes to growth.

Alterations in the intestinal microbial and viral communities are one marker of disease, however markers of inflammation are also important. An indirect measure of inflammation in the gastrointestinal tract is fecal calprotectin (FC), which is specific for neutrophils in the mucosa. Beaufils et al. ${ }^{19}$ found that FC level > $250 \mu \mathrm{g} / \mathrm{g}$ was associated with worse gastrointestinal symptoms and quality of life scores as measured by The Gastrointestinal Symptoms Scales 3.0-PedsQL ${ }^{\mathrm{TM}}$ and the Quality-of-Life Pediatric Inventory 4.0-PedsQL ${ }^{\mathrm{TM}}$. It can be speculated that in the future, a combination of fecal calprotectin, bacterial and viral microbiome assessment will provide a complete picture of an individual's intestinal milieu.

\section{LIVER}

Understanding liver disease in $\mathrm{CF}$ is important as there can be serious morbidity such as esophageal varices leading to hemorrhage and need for liver transplantation. An improved understanding of the epidemiology, various biomarkers, and novel testing modalities for liver disease in CF have been published this year.

PUSH (Prospective Study of Ultrasound to Predict Hepatic Cirrhosis in $\mathrm{CF}$ ) ${ }^{20}$, a nine year, multicenter, case-controlled, cohort study of children 3-12 years of age without known cirrhosis reported interim findings after 4 years. Participants $(n=55)$ with heterogeneous liver ultrasound were matched 1:2 to those with a normal ultrasound pattern $(\mathrm{n}=116)$. Biomarkers, GGT (gamma glutamyl transferase), AST (aspartate transaminase), ALT (alanine transaminase), platelets, AST to platelet ratio (APRI score) and Fibrosis Index based on four factors (age, platelet count, AST and ALT) (FIB-4) were significantly different at baseline in 
those with heterogeneous liver ultrasound. Of those with a heterogeneous liver ultrasound, a relative risk of $9.1(95 \%$ CI $2.7-30.8)$ was seen in developing a nodular ultrasound pattern in $13 \%(13 / 55)$ compared to 2.6 $\%(3 / 116)$ in those with a normal pattern.

As briefly outlined above, the PUSH study evaluated various laboratory markers, which other researchers have reported this year. Although the studies have varied methods and findings, GGT has been shown to be a key laboratory assessment. In a single center, retrospective, longitudinal study, Karnsakul et al. ${ }^{21}$ evaluated the potential predictivity for CFLD of numerous biomarkers: GGT alone, GGT to platelet ratio (GPR), AST/ALT ratio, APRI score, FIB-4, and platelet count. Cystic fibrosis liver disease (CFLD) was defined as objective evidence of advanced liver disease or cirrhosis on imaging, biopsy or portal hypertension $(\mathrm{PH})$. All of the biomarkers were predictive of CFLD except the FIB-4. GGT and GPR were noted to be representative of fibrosis, with GGT viewed specifically as a marker of early disease. With the inclusion of platelets alone or as part of GPR and APRI, a more sensitive prediction of advanced liver disease evidence of PH can be made. For example, patients with a GPR [?] 0.28 had 141.9 increased odds of developing CFLD. Further evaluation of biomarkers and their ability to characterize $\mathrm{PH}$ was conducted in a cohort of $577 \mathrm{CF}$ infants, diagnosed by NBS at two Italian CF centers, followed longitudinally through age 18.5 years $/ 2014^{59}$. $\mathrm{PH}$ was diagnosed by the presence of splenomegaly and confirmatory imaging. The overall incidence of $\mathrm{PH}$ was $8.8 \%$, with those with $\mathrm{PH}$ having a 4 times higher mortality/transplant risk. When analyzed as a whole to include all study sites or all individuals at a single study site, elevations on two or more occasions in GGT alone, as well as ALT alone, and combinations of elevations of ALT/GGT, GGT/ALP and ALT/ALP were predictive of the later development of $\mathrm{PH}$. However as less than $30 \%$ of patients with $\mathrm{PH}$ had any of these elevations, these parameters cannot be used on an individual patient basis to predict disease progression. All of these studies have shown the importance of GGT in predicting liver disease in CF either alone or in coordination with other biomarkers, highlighting the importance of obtaining a GGT when assessing liver enzymes.

Beyond biomarkers, there are many novel imaging modalities for CFLD that have been reported on this year. Calvopina et al. ${ }^{22}$ studied the potential of supersonic shear-wave elastography (SSWE) assessment of liver stiffness measurements (LSM) at detecting CFLD severity. CFLD was defined as the presence of at least two of three signs or symptoms: hepatomegaly \pm splenomegaly, persistent elevation (6 months) of ALT $(1.5 \times$ upper limit of normal ULN), or abnormal ultrasound scan (showing abnormal echogenicity or nodularity suggesting cirrhosis) ${ }^{23}$. Advanced CFLD was defined as CFLD with PH evidence by the presence of splenomegaly on ultrasound. SSWE determined LSM demonstrated good accuracy for detecting CLFD with LSM being higher in those with CFLD (8.1 kPa, IQR 6.7-11.9) compared those with CF but no liver diseases $(6.2 \mathrm{kPa}$, IWR $5.6-7.0, \mathrm{p}<0.0001)$. Interestingly those with $\mathrm{CF}$ and no liver disease were also able to be distinguished from healthy controls. In CF, a cut off of $6.85 \mathrm{kPa}$ had a sensitivity of $75 \%$ and a specificity of $71 \%$, with each $1 \mathrm{kPa}$ increase in LSM associated with a 1.7 -fold increase in odds of having CFLD. Finally, they also found a cut off of $9.05 \mathrm{kPa}$ was able to discriminate mild from advanced liver disease. The studies on CFLD in the past year have taken our understanding further which will help with advances in treatment. By understanding the potential markers of development of CFLD, caregivers can screen more effectively and counsel patients about their potential CFLD risks.

\section{Conclusion:}

Much has been learned in the past year's publications related to gastrointestinal disease and nutrition. The lessons from 2020 for nutrition include: 1) reminders to accurately assess pancreatic status via genotype/phenotype correlation; 2) apps can help optimize PERT dosing; 3) a new nutritional supplement can aid in fat absorption; 4) nutritional deficiencies are common; and 5) reinforcement that obesity is not uncommon in $\mathrm{CF}$ and is associated with a plateau effect on FEV1pp. The intestinal microbiome is altered early in CF life, with specific environmental modifiers such as environmental tobacco smoke exposure and antibiotics, leading to more pathogenic bacteria, while no microbial alterations are seen with acid blocking medications. Low length was shown to be associated with differences in the intestinal microbiome, allowing for the possibility of the intestinal microbiome treatment to improve growth. Liver disease severity can be assessed with 
biomarkers, with GGT being a key biomarker for severity, and ultrasound, with findings of a heterogenous liver being 9 times more likely to progress to nodular disease and liver stiffness can distinguish severity. It is our hope that this review helps advance clinicians understanding of research published in nutrition and the gastrointestinal involvement within $\mathrm{CF}$ published throughout the year.

\section{References:}

1. Poli P, Conforti C, Gennari E, Padoan R. Transitory pancreatic insufficiency in cystic fibrosis children. J Cyst Fibros 2020;19(1):e5-e6.

2. Boon M, Calvo-Lerma J, Claes I, Havermans T, Asseiceira I, Bulfamante A, Garriga M, Masip E, van Schijndel BAM, Fornes V, et al. Use of a mobile application for self-management of pancreatic enzyme replacement therapy is associated with improved gastro-intestinal related quality of life in children with Cystic Fibrosis. J Cyst Fibros 2020;19(4):562-568.

3. Calvo-Lerma J, Boon M, Colombo C, de Koning B, Asseiceira I, Garriga M, Roca M, Claes I, Bulfamante A, Walet S, et al. Clinical evaluation of an evidence-based method based on food characteristics to adjust pancreatic enzyme supplements dose in cystic fibrosis. J Cyst Fibros 2020;S1569-1993(20)30926-7.

4. Viola G, Mietto L, Secchi F, Ping L, Bruni A. Absorption and distribution of arachidonate in rats receiving lysophospholipids by oral route. J Lipid Res 1993;34(11):1843-52.

5. Stallings VA, Tindall AM, Mascarenhas MR, Maqbool A, Schall JI. Improved residual fat malabsorption and growth in children with cystic fibrosis treated with a novel oral structured lipid supplement: A randomized controlled trial. PLoS One 2020;15(5):1-13.

6. Tham A, Katz TE, Sutherland RE, Garg M, Liu V, Tong CW, Brunner R, Quintano J, Collins C, Ooi CY. Micronutrient intake in children with cystic fibrosis in Sydney, Australia. J Cyst Fibros 2020;19(1):146-152.

7. Harindhanavudhi T, Wang Q, Dunitz J, Moran A, Moheet A. Prevalence and factors associated with overweight and obesity in adults with cystic fibrosis: A single-center analysis. J Cyst Fibros 2020;19(1):139145.

8. Lahiri T, Sullivan JS, Sartorelli KH, Murphy JJ. Delayed Presentation of Meconium Ileus in an Infant With Cystic Fibrosis. Pediatrics 2020;146(4).

9. Christensen C, Sivapatham G, McKinney ML, Stammers D. Sulfhemoglobinemia associated with meconium ileus in cystic fibrosis. Pediatr Pulmonol 2020;55(10):2496-2497.

10. Morales Morales CA, Gonzalez-Urquijo M, Morales Flores LF, Quevedo-Fernandez E, Guzmán Huerta EA, Virgilio Hernández-Torre MM. Proximal intestinal obstruction syndrome (PIOS) in a patient with cystic fibrosis: A case report. Ann Med Surg 2020;60(December):669-672.

11. Klouda TM, Lindholm E, Poletto E, Rani S, Varlotta L, Velasco J. Presentation of an H-type tracheoesophageal fistula in an adolescent male with cystic fibrosis: A case report and review of literature. Clin Imaging 2020;60(1):38-47.

12. Kuczynski J, Liu Z, Lozupone C, Mcdonald D, Fierer N, Knight R. To Detect Biologically Relevant Patterns. Nat Methodsat Methods 2010;7(10):813-819.

13. Lozupone CA, Knight R. Species divergence and the measurement of microbial diversity. FEMS Microbiol Rev 2008;32(4):557-578.

14. Kristensen M, Prevaes SMPJ, Kalkman G, Tramper-Stranders GA, Hasrat R, de Winter- de Groot KM, Janssens HM, Tiddens HA, van Westreenen M, Sanders EAM, et al. Development of the gut microbiota in early life: The impact of cystic fibrosis and antibiotic treatment. J Cyst Fibros 2020;19(4):553-561.

15. Khalaf RT, Furura GT, Wagner BD, Roberson CE, Andrews R, Stevens MJ, Fillon SA, Zemanick ET HJ. Influence of acid blockade on the aerodigestive tract microbiome in children with cystic fibrosis. J Pediatr 
Gastroenterol Nutr 2021;72(4):520-527.

16. Loman BR, Shrestha CL, Thompson R, Groner JA, Mejias A, Ruoff KL, O’Toole GA, Bailey MT, Kopp BT. Age and environmental exposures influence the fecal bacteriome of young children with cystic fibrosis. Pediatr Pulmonol 2020;55(7):1661-1670.

17. Hayden HS, Eng A, Pope CE, Brittnacher MJ, Vo AT, Weiss EJ, Hager KR, Martin BD, Leung DH, Heltshe SL, et al. Fecal dysbiosis in infants with cystic fibrosis is associated with early linear growth failure. Nat Med 2020;26(2):215-221.

18. Coffey MJ, Low I, Stelzer-Braid S, Wemheuer B, Garg M, Thomas T, Jaffe A, Rawlinson WD, Ooi CY. The intestinal virome in children with cystic fibrosis differs from healthy controls. PLoS One 2020;15(5):1-18.

19. Beaufils F, Mas E, Mittaine M, Addra M, Fayon M, Delhaes L, Clouzeau H, Galode F, Lamireau T. Increased Fecal Calprotectin Is Associated with Worse Gastrointestinal Symptoms and Quality of Life Scores in Children with Cystic Fibrosis. 2020:1-14.

20. Siegel MJ, Freeman AJ, Ye W, Palermo JJ, Molleston JP, Paranjape SM, Stoll J, Leung DH, Masand P, Karmazyn B, et al. Heterogeneous Liver on Research Ultrasound Identifies Children with Cystic Fibrosis at High Risk of Advanced Liver Disease: Interim Results of a Prospective Observational Case-Controlled Study. J Pediatr 2020;219:62-69.e4.

21. Karnsakul W, Wasuwanich P, Ingviya T, Vasilescu A, Carson KA, Mogayzel PJ, Schwarz KB. A longitudinal assessment of non-invasive biomarkers to diagnose and predict cystic fibrosis-associated liver disease. J Cyst Fibros 2020;19(4):546-552.

22. Calvopina DA, Noble C, Weis A, Hartel GF, Ramm LE, Balouch F, Fernandez-Rojo MA, Coleman MA, Lewindon PJ, Ramm GA. Supersonic shear-wave elastography and APRI for the detection and staging of liver disease in pediatric cystic fibrosis. J Cyst Fibros 2020;19(3):449-454.

23. Debray D, Kelly D, Houwen R, Strandvik B, Colombo C. Best practice guidance for the diagnosis and management of cystic fibrosis-associated liver disease. J Cyst Fibros 2011;10(SUPPL. 2):S29-S36.

\section{Hosted file}

Table 1 Part 3B.docx available at https://authorea.com/users/418014/articles/524898-year-inreview-2020-nutrition-and-gastrointestinal-disease-in-cystic-fibrosis 